\title{
QUAL A MELHOR CONDUTA TERAPÊUTICA NÃO-MEDICAMENTOSA PARA PACIENTES COM DOENÇA HEPÁTICA GORDUROSA NÃO-ALCOÓLICA?
}

\author{
Alan José Barbosa Magalhães ${ }^{1}$, Regina Celi Trindade Camargo ${ }^{2}$, Robson Chacon Castoldi ${ }^{3,4}$, \\ Guilherme Akio Tamura Ozaki², Tatiana Emy Koike², Thiago Alves Garcia², Vinícius Gustavo \\ Gimenes Turato ${ }^{2}$, Elaine Aparecida Lozano da Silva², José Carlos Silva Camargo Filho \\ Universidade Estadual Paulista - FCT/UNESP, ${ }^{1}$ Especialização em Ortopedia e Traumatologia, ${ }^{2}$ Departamento de \\ Fisioterapia, Presidente Prudente, SP. 3Universidade do Oeste Paulista - UNOESTE, Curso de Educação Física, \\ Presidente Prudente, SP. ${ }^{4}$ Universidade Estadual de Campinas - UNICAMP, Faculdade de Ciências Médicas, SP. e-mail: \\ ajb magalhaes@yahoo.com.br
}

\section{RESUMO}

Estudos mostram um crescente aumento no número de pessoas com excesso de peso no Brasil. Dentre outros fatores prejudiciais pelo excesso de peso, a Doença Hepática Gordurosa NãoAlcoólica (DHGNA) pode ser seriamente prejudicial à saúde. Esta revisão sistemática tem por objetivo verificar qual a melhor conduta não-medicamentosa para pacientes com DHGNA. Foi realizada uma busca nas bases de dados BIREME, MEDLINE, PEDro, PubMed e SciELO, e o período de publicação dos estudos foi de 2008 à 2013. Dentre os artigos encontrados, foi realizada uma análise nos títulos e resumos, visando confirmar se os artigos se encontravam nos critérios de seleção estabelecidos; os estudos deveriam ser originais; não deveriam ser revisões sistemáticas. Os achados mostraram que a utilização de uma dieta adequada e exercícios físicos são eficazes em pacientes com DHGNA, mas novos estudos são necessários para padronizar a conduta para estes pacientes.

Palavras-chave: dieta, exercício, fígado, fígado gorduroso, obesidade.

\section{WHAT IS THE BEST NON-DRUG TREATMENT FOR PATIENTS WITH NON-ALCOHOLIC FATTY LIVER DISEASE?}

\begin{abstract}
Studies show a steady increase in the number of overweight people in Brazil. Among other detrimental factors for overweight, the Non-alcoholic fatty liver (NAFLD) disease can be seriously harmful to health. This systematic review aims to determine what the best nonm-drug approach for patients with NAFLD. A search was conducted on the basis of BIREME, MEDLINE, PEDro, PubMed and SciELO data and the publication period of study was 2008 to 2013. Among the articles found, an analysis was performed in the titles and abstracts, aiming to confirm whether the items met the selection criteria established; studies should be original; should not be systematic reviews. The results show that the use of a proper diet and exercise are effective in patients with NAFLD, but further studies are needed to standardize the best treatment for these patients.
\end{abstract}

Keywords: diet, exercise, liver, fatty liver, obesity. 


\section{INTRODUÇÃO}

Se comparados os resultados da Pesquisa de Orçamentos Familiares (POF) de 2002-2003 com 2008-2009, verifica-se que a frequência de pessoas com excesso de peso no Brasil aumentou em mais de um ponto percentual ao ano (homens de $41,4 \%$ para $50,1 \%$ e mulheres de $40,9 \%$ para $48,0 \%$, respectivamente), o que indica que, em cerca de dez anos, o excesso de peso poderia alcançar dois terços da população adulta do Brasil. Magnitude esta idêntica à encontrada na população dos Estados Unidos ${ }^{1}$. A obesidade afeta um terço da população dos Estados Unidos e um quinto da população europeia ${ }^{2}$.

Em pessoas obesas, um aumento na concentração de triglicerídeos intrahepáticos (TGIH) pode ser um indício de síndrome metabólica, independente do Índice de Massa corporal (IMC) ou índice de gordura corporal do indivíduo ${ }^{3}$. O tecido adiposo visceral desempenha um papel importante na liberação de citocinas e mediadores bioativos ${ }^{4}$. Seu acúmulo excessivo pode ser prejudicial à saúde.

O fígado possui um papel importante no metabolismo lipídico, desde o armazenamento de ácidos graxos da dieta pela secreção de lipoproteínas até sua oxidação, para a produção de energia para todo o corpo ${ }^{5}$.
A obesidade está associada a uma gama de anormalidades por meio de infiltração gordurosa no fígado de crianças e adultos, conhecida como Doença Hepática Gordurosa Não Alcoólica (DHGNA) ${ }^{6}$.

A DHGNA é caracterizada pelo acúmulo excessivo de lipoproteínas de baixa intensidade (VLDL) e triglicerídeos séricos (TG) no fígado ${ }^{3,7}$, podendo ser uma manifestação da síndrome metabólica e especificamente relatada por obesidade e resistência à insulina ${ }^{6,8}$. A manifestação da DHGNA pode variar desde esteatose à esteatohepatite não-alcoólica (EHNA), podendo progredir à cirrose, fibrose e hepatocarcinoma celular 9 .

Do ponto de vista químico, a EHNA é definida quando o TGIH representa $5 \%$ do peso ou volume total do órgão ${ }^{10}$. Do ponto de vista histológico, é definida quando $5 \%$ ou mais dos hepatócitos possuem triglicerídeo intracelular, além de inflamação lobular, esteatose microvesicular ou macrovesicular (variando com o tamanho dos vacúolos lipídicos) e degeneração hepatocelular em baloneamento $^{11,12} \mathrm{com}$ ou sem corpos de Mallory, além de inflamação portal ou lobular, com ou sem fibrose ${ }^{10}$.

Indivíduos com esteatose hepática somada à lesão tecidual, morte celular e alterações teciduais provenientes do processo inflamatório possuem mais chances de desenvolver cirrose, fibrose e câncer do 
que indivíduos que apresentam estestose simples ${ }^{13,14}$.

Finucane (2010) mostra que pacientes com esteatose simples apresentam 1-2\% de risco de desenvolver cirrose em 15-20 anos, mas pacientes com EHNA possuem risco de $12 \%$ de desenvolver a doença em oito anos. Além disso, estes pacientes possuem uma alta predisposição ao desenvolvimento de doenças cardiovasculares ${ }^{12}$.

A diminuição no peso corporal gradual exerce papel importante não só na melhora do quadro do paciente, como também na prevenção de futuras doenças hepáticas ${ }^{16}$. O exercício deve ser rotineiro para portadores da doença, pois estas intervenções podem reduzir os níveis de TGIH, podendo refletir ou não perda de peso $^{17}$.

Do Valle ${ }^{18}$ verificou que a prática de exercícios reduz os níveis de TG e aumenta o HDL-c (lipoproteína de alta intensidade). Além disso, um programa de treinamento moderado à vigoroso pode gerar diminuição nas concentrações de tecido adiposo visceral ${ }^{2}$.

Tendo em vista o conteúdo apresentado, esta revisão sistemática tem por objetivo verificar qual a melhor conduta não-medicamentosa para pacientes com DHGNA.

\section{MATERIAIS E MÉTODOS}

A revisão sistemática da literatura foi realizada apenas em base de dados eletrônica, em função da melhor acessibilidade e gratuidade dos trabalhos acadêmicos em relação às vias impressas. A pesquisa foi realizada somente nas bases de dados da BIREME, MEDLINE, PEDro, PubMed e SCIELO.

Para o processo de busca, foram utilizadas as seguintes palavras-chave: diet, exercise, fatty liver, liver, obesity. Os descritores foram pesquisados nos idioma inglês, tendo em vista encontrar uma maior quantidade de trabalhos atuais. Foram pesquisados trabalhos datados entre janeiro de 2008 a dezembro de 2013.

A seleção dos trabalhos foi realizada da seguinte forma: dentre os artigos encontrados, foi realizada uma análise nos títulos e resumos, visando confirmar se os artigos se encontravam nos critérios de seleção estabelecidos; os estudos deveriam ser originais; não deveriam ser revisões sistemáticas. Em seguida, os artigos eram obtidos na íntegra e examinado se possuíam análises independentes (sexo, idade e finalidade do estudo: estado de saúde e tratamento).

Após a realização da pesquisa, foram encontrados 40 trabalhos na íntegra, onde 15 foram realizados com ratos, dois estavam fora do escopo predefinido e oito eram 
revisões sistemáticas. Todos os estes trabalhos encontrados que não correspondiam com o objetivo da pesquisa inicial foram excluídos, ficando apenas 15 estudos clínicos randomizados originais. Uma planilha inicial foi montada, com informações relevantes sobre a metodologia de cada estudo, tanto dos artigos incluídos no estudo como dos trabalhos que não foram adicionados à revisão.

Para comparação estatística dos resultados, foi utilizado o Teste Qui-
Quadrado, para comparar proporções. Todos os procedimentos adotaram o valor de significância de $(p<0,05)$. Os cálculos foram realizados com o aplicativo SPSS 17.0 for Windows ${ }^{\circledR}$.

\section{RESULTADOS}

Dentre os 15 trabalhos selecionados, todos foram publicados no intervalo de 2008 a 2013 (Tabela 1).

Tabela 1. Características dos estudos abordados.

\begin{tabular}{|c|c|c|c|c|}
\hline Autor & Periódico & Ano & Intervenção & Sexo* \\
\hline Bacchi et $a l^{30}$ & Hepatology & 2013 & Exercício & $\mathrm{M} / \mathrm{F}$ \\
\hline Eckard et al 22 & Therap Adv Gastroenterol & 2013 & Dieta e Exercício & M \\
\hline George et $a^{23}$ & J Gastroen Hepatol & 2009 & Dieta e Exercício & $M$ \\
\hline Hallsworth et al ${ }^{32}$ & Hepatology & 2011 & Exercício & $\mathrm{M} / \mathrm{F}$ \\
\hline Jakovljevic et $a l^{31}$ & Clin Sci & 2013 & Exercício & $\mathrm{M} / \mathrm{F}$ \\
\hline Kistler et $a l^{33}$ & Am J Gastroenterol & 2011 & Exercício & $M / F$ \\
\hline Lee et $a l^{27}$ & $\begin{array}{l}\text { Am J Physiol Endocrinol } \\
\text { Metab }\end{array}$ & 2013 & Exercício & $\mathrm{F}$ \\
\hline Piano et $a l^{20}$ & Eur J Gastroen Hepat & 2012 & Exercício & $\mathrm{M} / \mathrm{F}$ \\
\hline Promrat et al ${ }^{24}$ & Hepatology & 2010 & Dieta e Exercício & $\mathrm{M} / \mathrm{F}$ \\
\hline Pugh et $a l^{26}$ & $\begin{array}{l}\text { Am J Physiol Endocrinol } \\
\text { Metab }\end{array}$ & 2013 & Exercício & $\mathrm{M} / \mathrm{F}$ \\
\hline Shah et al ${ }^{25}$ & Obesity & 2009 & Dieta e Exercício & $\mathrm{F}$ \\
\hline Slentz et $a l^{29}$ & $\begin{array}{l}\text { Am J Physiol Endocrinol } \\
\text { Metab }\end{array}$ & 2011 & Exercício & $\mathrm{M} / \mathrm{F}$ \\
\hline Straznicky et al ${ }^{19}$ & Diabetes Obes Metab & 2012 & Dieta e Exercício & $\mathrm{M} / \mathrm{F}$ \\
\hline Sullivan et $a l^{28}$ & Hepatology & 2012 & Exercício & $\mathrm{M} / \mathrm{F}$ \\
\hline Wang et $a l^{21}$ & World J Gastroentero & 2008 & Dieta e Exercício & $\mathrm{M} / \mathrm{F}$ \\
\hline
\end{tabular}

Nota-se que a maioria dos estudos foi realizada com um grupo amostral de ambos os sexos $(73,4 \%)$, seguido de grupos compostos apenas por mulheres (13,3\%) e homens $(13,3 \%)$.

Nestes trabalhos, também houve maior número de estudos com intervenções 
relacionados ao exercício (56,3\%), seguido de dieta e exercício $(43,7 \%)$

Dentre os tipos de treinamento encontrado em ambas intervenções, o treinamento aeróbio foi o mais utilizado $(68,8 \%)$, seguido do concorrente (treinamento aeróbio + treinamento de resistência; $18,7 \%$ ) e anaeróbio (12,5\%).

\section{DISCUSSÃO}

No período de 2008 a 2013, foram encontrados 40 trabalhos, onde apenas 15 eram ensaios clínicos randomizados originais, o que demonstra a escassez de estudos na área. Além disso, a variedade de intervenções propostas e tipos de exercícios utilizados apontam para uma dificuldade de se chegar a um consenso que possa protocolar o tratamento de portadores dessa patologia.

Dentre os trabalhos encontrados, foi proposta por todos os autores a adesão à prática de exercícios físicos para a diminuição de concentração de lipídios no tecido hepático dos pacientes, ficando ainda sem consenso quanto à intensidade dos exercícios. Há ainda controvérsias quanto ao uso ou não de uma dieta adequada associada à prática de exercícios físicos.

Estudos apontam que em sujeitos obesos que perderam peso $(8-9 \%$ do peso corporal) com ou sem treinamento aeróbico houve uma melhora da função das enzimas hepáticas, regulando assim o metabolismo corporal, pois a perda de peso ocasiona redução da massa gorda abdominal e da gordura saturada circulante no corpo ${ }^{19}$.

Tendo isso em vista, uma terapia interdisciplinar em longo prazo somada a um protocolo de treinamento concorrente, é eficaz para melhorar os níveis de esteatose hepática ${ }^{20}$, pois a prática de exercícios somada a uma nutrição equilibrada e adequada traz melhora na resposta à insulina e função hepática de pacientes com DHGNA $^{21}$. Um estudo ${ }^{22}$ verificou que mudanças no estilo de vida de pacientes (pratica de exercícios físicos e realização de dieta adequada, com baixas calorias) com esteatohepatite não alcoólica e DHGNA por pelo menos 6 meses, gera melhoras na histologia hepática, em função da perda de peso gerada pela intervenção (perda média de 1,1 - 2,9 Kg no protocolo proposto).

Outro estudo observou que este tipo de intervenção pode também proporcionar melhora nas funções de enzimas hepáticas e regulação do metabolismo ${ }^{23}$, perda de peso de $7 \%-10 \%$, diminuição na esteatohepatite em pacientes que seguiram o mesmo estilo de vida ${ }^{24}$, além de diminuição de aproximadamente $50 \%$ de gordura intrahepática, além de melhora da resposta à insulina de aproximadamente $60 \%$, com diminuição de desenvolvimento de diabetes tipo II nestes mesmos pacientes ${ }^{25}$. 
Também foram propostos diversos tipos de treinamentos que demonstraram ser eficazes no tratamento físico de pacientes com DHGNA. No entanto, há controversa quanto ao tipo de treinamento mais indicado para este caso em específico. Alguns achados verificaram que um treinamento aeróbio melhora a resposta microvascular de pacientes com DHGNA, reduzindo também os riscos de doenças cardiovasculares ${ }^{26}$, além de uma redução na quantidade de gordura intramuscular, gordura visceral, gordura intrahepática e melhora na resposta à insulina em pacientes obesos ${ }^{27}$.

Um estudo verificou que um treinamento aeróbio de intensidade moderada tem efeitos modestos sobre o TG hepático e metabolismo de lipoproteínas, com redução leve no teor de TGIH e ácidos graxos livres (AGL), mas sem alterar taxas de secreção hepática de VLDL-TG ou VLDL-apoB100 ou as concentrações plasmáticas de lipídios em pacientes com DHGNA ${ }^{28}$. No entanto, a adição de um treinamento de resistência para estes pacientes não produz alterações significativas nos resultados já conquistados $^{29}$.

Porém, alguns autores defenderam o uso do treinamento concorrente ou de resistência (isolado e treinamento aeróbio) no tratamento de pacientes com DHGNA. Sugere-se que um programa de quatro meses de treinamento concorrente é igualmente eficaz para redução de gordura hepática em pacientes sedentários com diabetes tipo II DHGNA $^{30}$, melhorando também a resposta simpatovagal, variabilidade da frequência cardíaca, resposta dos baroceptores e respostas hemodinâmicas destes pacientes ${ }^{31}$.

Exercícios de resistência bem tolerados reduzem o teor de $\mathrm{TGIH}$, melhoram a resposta à insulina e a flexibilidade metabólica de pacientes com DHGNA, independente de ocorrer perda de peso ou não dos pacientes durante o período da aplicação de protocolo, onde os efeitos deste tipo de exercício são semelhantes aos exercícios aeróbios, onde tais benefícios podem ou não ser potencializados caso haja também um acompanhamento a longo prazo da terapêutica somada à restrição calórica adequada, sem a adição de nenhuma intervenção adicional ${ }^{32}$.

Houve ainda um estudo que discorda do que já foi apresentado, pois verificou uma relação inversa entra prática de atividades físicas vigorosas e DHGNA, onde atividades de intensidade moderada podem não ser eficazes para redução dos níveis de lipídios no fígado ${ }^{33}$.

Não houve consenso entre os estudos encontrados em relação ao melhor tipo de treinamento a ser utilizado, melhor período de duração, melhor tipo de dieta a ser empregada e da necessidade de se utilizar apenas exercícios físicos no tratamento ou da 
utilização em conjunto com uma dieta adequada. Por ser evidenciada por meio deste estudo a escassez na literatura quanto a estudos que estabeleçam a melhor intervenção para pacientes com DHGNA, acredita-se que há a necessidade de mais ensaios clínicos randomizados nesta área, visando estabelecer um consenso quanto a melhor conduta a ser realizada. O presente estudo também possui algumas limitações, pois se restringiu a pesquisar artigos originais de ensaios clínicos randomizados apenas nas bases de dados apresentadas, com as palavras-chave utilizadas, apenas no idioma inglês.

Em vista do que foi apresentado, conclui-se que a prática de exercícios físicos somada ou não a uma dieta adequada traz resultados positivos no tratamento de pacientes com DHGNA. Entretanto, há a necessidade da realização de mais estudos para que se possa estabelecer qual tipo de exercício é o melhor, se deve ser utilizado de maneira isolada ou não a outra conduta terapêutica, bem como o melhor tipo de dieta e tempo de tratamento.

\section{CONFLITO DE INTERESSES}

Os autores declaram não haver qualquer potencial conflito de interesses que possa interferir na imparcialidade deste trabalho científico.

\section{REFERÊNCIAS}

1. IBGE. Pesquisa de Orçamentos Familiares (POF) 2008-2009. Disponível em: <http://www.ibge.gov.br/home/presidencia/ noticias/noticia_visualiza.php?id_noticia=169 9\&id_pagina=1>. Acessado em 01/07/2014.

2. Vissers D, Hens W, Taeymans J, Baeyens J, Poortmans J, Gaal LV. The Effect of Exercise on Visceral Adipose Tissue in Overweight Adults: A Systematic Review and MetaAnalysis. Plos One. 2013;8(2):1-10. DOI: http://dx.doi.org/10.1371/journal.pone.0056 $\underline{415}$

3. Fabbrini E, Mohammed BS, Magkos F, Korenblat KM, Patterson BW, Klein S. Alterations in adipose tissue and hepatic lipid kinetics in obese men and women with nonalcoholic fatty liver disease. Gastroenterology. 2008;134:424-431. DOI: http://dx.doi.org/10.1002/hep.23280

4. Ahima RS. Adipose tissue as an endocrine organ. Obesity (Silver Spring). 2006;14(Suppl 5):242S-249S.

DOI: http://dx.doi.org/10.1016/S10432760(00)00301-5

5. Fritsche L, Weigert C, Häring HU, Lehmann R. How insulin receptor substrate proteins regulate the metabolic capacity of the liver implications for health and disease. Curr Med Chem. 2008;15:1316-1329. DOI: http://dx.doi.org/10.2174/092986708784534 $\underline{956}$

6. Moore J. Non-alcoholic fatty liver disease: the hepatic consequence of obesity and the metabolic syndrome. Proc Nutr Soc. 2010;69:211-220. DOI: http://dx.doi.org/10.1017/S00296651100000 $\underline{30}$

7. Adiels M, Taskinen MR, Packard C, Caslake $M$, Soro-Paavonen A, Westerbacka J et al. Overproduction of large VLDL particles is driven by increased liver fat content in man. Diabetologia. 2006;49:755-765. DOI: 
http://dx.doi.org/10.1007/s00125-005-0125$\underline{z}$

8. Angulo P. Gl epidemiology: nonalcoholixc fatty liver disease. Aliment Pharmacol Therapeut. 2007;25:883-889. DOI: http://dx.doi.org/10.1111/j.1365$\underline{2036.2007 .03246 . x}$

9. Loomba R, Sirlin CB, Schwimmer JB, Lavine JE. Advances in Pediatric Nonalcoholic Fatty Liver Disease. Hepatology. 2009;50(4):12821293.

DOI: http://dx.doi.org/10.1002/hep.23119

10. Kleiner DE, Brunt EM, Van Natta $M$, Behling C, Contos MJ, Cummings OW, Ferrell LD et al. Design and validation of a histological scoring system for nonalcoholic fatty liver disease. Hepatology. 2005;41:1313-1321. DOI:

http://dx.doi.org/10.1002/hep.20701

11. Kneeman JM, Misdraji J, Corey KE. Secondary causes of nonalcoholic fatty liver disease. Ther Adv Gastroenterol. 2012;5(3):199-207. DOI: http://dx.doi.org/10.1177/1756283X1143085 $\underline{9}$

12. Misra VL, Khashab M, Chalasani N. NonAlcoholic Fatty Liver Disease and Cardiovascular Risk. Curr Gastroenterol Rep. 2009;11(1):50-55. DOI: http://dx.doi.org/10.1007/s11894-009-0008$\underline{4}$

13. Syn WK, Teaberry V, Choi SS et al. Similarities and differences in the pathogenesis of alcoholic and nonalcoholic steatohepatitis. Semin Liver Dis. 2009;29:200-10. DOI: http://dx.doi.org/10.1136/gutjnl-2011$\underline{301857}$

14. Adams LA, Lymp JF, St Sauver J et al. The natural history of nonalcoholic fatty liver disease: a population-based cohort study. Gastroenterology. 2005;129:113-21. DOI: http://dx.doi.org/10.1053/i.gastro.2005.04.0 $\underline{14}$

15. Finucane $F$, Sharp S, Purslow L et al. The effects of aerobic exercise on metabolic risk, insulin sensitivity and intrahepatic lipid in healthy older people from the Hertfordshire Cohort Study: a randomised controlled trial. Diabetologia. 2010;53:624-31. DOI: http://dx.doi.org/10.1007/s00125-009-1641$\underline{z}$

16. Duvnjak M, Tomasic V, Gomercic $M$, Duvnjak LS, Barsic N, Lerotic I. Therapy of Nonalcoholic Fatty Liver Disease: Current Status. J Physiol Pharmacol. 2009;60(Suppl 7):57-66.

17. Keating SE, Hackett DA, George J, Johnson NA. Exercise and non-alcoholic fatty liver disease: A systematic review and metaanalysis. J Hepatol. 2012;57:157-166. DOI: http://dx.doi.org/10.1016/i.jhep.2012.02.023

18. Do Valle VS, Mello DB, Fortes MSR, Dantas EHM, Mattos MA. Efeito da Dieta e do Ciclismo Indoor Sobre a Composição Corporal e Nível Sérico Lipídico. Arq Bras Cardiol. 2010;95(2):173-8.

DOI: http://dx.doi.org/10.1590/S1415-

$\underline{52732010000600003}$

19. Straznicky NE, Lambert EA, Grima MT, Eikelis N, Nestel PJ, Dawood T et al. The effects of dietary weight loss with or without exercise training on liver enzymes in obese metabolic syndrome subjects. Diabetes Obes Metab. 2012:14:139-148. DOI: http://dx.doi.org/10.1111/i.1463-

1326.2011.01497.x

20. Piano A, Mello MT, Sanches PL, Silva PL, Campos RMS, Carnier J et al. Long-term effects of aerobic plus resistance training on the adipokines and neuropeptides in nonalcoholic fatty liver disease obese adolescents. Eur J Gastroen Hepat. 2012;24(11)1313-1324.
DOI: 
http://dx.doi.org/10.1097/MEG.0b013e3283 $\underline{5793 a c}$

21. Wang C, Liang L, Fu J, Zou C, Hong F, Xue J et al. Effect of lifestyle intervention on nonalcoholic fatty liver disease in Chinese obese children. World J Gastroenterol. 2008;14(10):1598-1602.

DOI:

http://dx.doi.org/10.3748/wjg.14.159

22. Eckard C, Cole R, Lockwood J, Torres DM, Williams CD, Shaw JC et al. Prospective histopathologic evaluation of lifestyle modification in nonalcoholic fatty liver disease: a randomized trial. Ther Adv Gastroenterol. 2013;6:249-259. DOI: http://dx.doi.org/10.1177/1756283X1348407 $\underline{8}$

23. George AS, Bauman A, Johnston A, Farrell G, Chey T, George J. Effect of a lifestyle intervention in patients with abnormal liver enzymes and metabolic risk factors. J Gastroen Hepatol. 2009;24:399-407. DOI: http://dx.doi.org/10.1111/i.1440-

\section{$\underline{1746.2008 .05694 . x}$}

24. Promrat K, Kleiner DE, Niemeier HM, Jackvony $E$, Kearns $M$, Wands JR et al. Randomized Controlled Trial Testing the Effects of Weight Loss on Nonalcoholic Steatohepatitis. Hepatology. 2010;51(1):121129.

DOI:

http://dx.doi.org/10.1002/hep.23276

25. Shah K, Stufflebam A, Hilton TN, Sinacore DR, Klein S, Villareal DT. Diet and Exercise Interventions Reduce Intrahepatic Fat content and Improve Insulin Sensitivity in Obese Older Adults. Obesity. 2009;17(12):2162-2168. DOI: http://dx.doi.org/10.1038/oby.2009.126

26. Pugh JAC, Cuthbertson DJ, Sprung VS, Kemp GJ, Richardson P, Umpleby AM et al. Exercise training improves cutaneous microvascular function in nonalcoholic fatty liver disease. Am J Physiol Endocrinol Metab. 2013;305:E50-E58. http://dx.doi.org/10.1152/ajpendo.00055.20 $\underline{13}$

27. Lee $S$, Deldin AR, White D, Kim Y, Libman I, Rivera-Vega $M$ et al. Aerobic exercise but not resistance exercise reduces intrahepatic lipid content and visceral fat and improves insulin sensitivity in obese adolescent girls: a randomized controlled trial. Am J Physiol Endocrinol Metab. 2013;305:E1222-E1229 DOI:

http://dx.doi.org/10.1152/ajpendo.00285.20 $\underline{13}$

28. Sullivan S, Kirk EP, Mittendorfer B, Patterson BW, Klein S. Randomized Trial of Exercise Effect on Intrahepatic Triglyceride Content and Lipid Kinetics in Nonalcoholic Fatty Liver Disease. Hepatology. 2012;55(6):1738-1745. DOI: http://dx.doi.org/10.1002/hep.25548

29. Slentz CA, Bateman LA, Willis LH, Shields AT, Tanner CJ, Piner LW et al. Effects of aerobic vs. resistance training on visceral and liver fat stores, liver enzymes, and insulin resistance by HOMA in overweight adults from STRRIDE AT/RT. Am J Physiol Endocrinol Metab. 2011;301:E1033-E1039. DOI: http://dx.doi.org/10.1152/ajpendo.00291.20 $\underline{11}$

30. Bacchi E, Negri C, Targher G, Faccioli N, Lanza $M$, Zoppini $G$ et al. Both Resistance Training and Aerobic Training Reduce Hepatic Fat Content in Type 2 Diabetic Subjects With Nonalcoholic Fatty Liver Disease (the RAED2 Randomized Trial). Hepatology. 2013;58(4):1287-1295. DOI: http://dx.doi.org/10.1002/hep.26393

31. Jakovljevic DG, Hallsworth K, Zalewski P, Thoma C, Klawe JJ, Day CP. Resistance Exercise Improves autonomic regulation at rest and haemodynamic response to exercise in non-alcoholic fatty liver disease. Clin Sci. 2013;125:143-149.

DOI: 
32. Hallsworth K, Fattakhova G, Hollingsworth KG, Thoma C, Moore S, Taylor R. Resistance exercise reduces liver fat and its mediators in non-alcoholic fatty liver disease independent of weight loss. Hepatology. 2011;60:1278-1283.

DOI:

http://dx.doi.org/10.1136/gut.2011.242073

33. Kistler KD, Brunt EM, Clark JM, Diehl AM, Sallis JF, Schwimmer JB. Physical Activity Recommendations, Exercise Intensity, and Histological Severity of Nonalcoholic Fatty Liver Disease. Am J Gastroenterol. 2011;1:19.

DOI: http://dx.doi.org/10.1038/ajg.2010.488

Recebido para publicação em 08/08/2014

Revisado em 20/03/2015

Aceito em 15/05/2015 